\title{
Functional Recovery Following Physical Training in Tracheotomized and Chronically Ventilated Patients
}

\author{
Enrico M Clini MD, Ernesto Crisafulli MD PhD, Francesca Degli Antoni, Claudio Beneventi, \\ Ludovico Trianni MD, Stefania Costi, Leonardo M Fabbri MD, and Stefano Nava MD
}

\begin{abstract}
BACKGROUND: Rehabilitation is a non-pharmacologic therapy that can restore health and reverse the patient's disability, but the efficacy of rehabilitation in critically ill patients is not well documented. METHODS: In a prospective cohort study, we assessed whether the degree of change in functional status after comprehensive rehabilitation influenced clinical outcomes in $\mathbf{7 7}$ tracheotomized patients (mean \pm SD age $75 \pm 7 \mathrm{y}$ ) admitted for difficult weaning to our regional weaning center. The care plan, including peripheral muscle training, was delivered daily. We recorded admission demographic, anthropometric, and functional characteristics. We measured the change in basic activities of daily living score $(\triangle \mathrm{BADL})$, survival, and weaning success rate as clinical outcomes. We performed the Pearson correlation analysis and linear regression, with $\triangle B A D L$ as the dependent variable, to test the predictive power of the baseline measurements. RESULTS: Sixty-seven patients (87\%) survived, and 55 of them (74\%) succeeded in weaning during their stay in the weaning center. The mean \pm SD $\triangle B A D L$ improvement was $2.5 \pm 2.0$ points (median 2 points). Baseline performance of the latissimus dorsi predicted $\triangle B A D L(\beta=0.388,95 \%$ CI $0.111-1,664, P=.03)$. The probability of remaining ventilator-free $(P=.043)$ and survival $(P=.001)$ differed across the $4 \Delta B A D L$ categories $(0=$ no change, $1-2=$ least improvement, and $>2=$ improvement above median change). CONCLUSIONS: Mortality rate and weaning success differ according to $\triangle \mathrm{BADL}$ following active rehabilitation/training in tracheotomized, ventilated, difficult-to-wean patients. The performance of the latissimus dorsi was the only significant predictor of change. Key words: tracheotomy; rehabilitation; muscle training; mechanical ventilation; weaning; activities of daily living. [Respir Care 2011;56(3):306-313. (c) 2011 Daedalus Enterprises]
\end{abstract}

\section{Introduction}

Weaning centers and respiratory intensive care units (ICUs) have been developed as hospital areas in which critically ill patients under prolonged mechanical ventilation are often admitted and cared for. ${ }^{1}$ Indeed, this condition may expose individuals in conventional ICUs to a higher risk of early mortality ${ }^{2,3}$ and late pulmonary complications, ${ }^{4}$ at greater expense. ${ }^{5}$

Enrico M Clini MD, Ernesto Crisafulli MD PhD, Francesca Degli Antoni, Claudio Beneventi, and Ludovico Trianni MD are affiliated with the Department of Pulmonary Rehabilitation, Ospedale Villa Pineta, Pavullo, Italy. Enrico M Clini MD, Stefania Costi, and Leonardo M Fabbri MD are affiliated with the Department of Oncology, Haematology and Respiratory Diseases, University of Modena-Reggio Emilia, Modena, Italy. At the time of this study Stefano Nava MD was affiliated with the Division of Pneumonology and the Intensive Care Unit, Fondazione Salvatore Maugeri, Istituto Di Ricovero e Cura
The chronically ventilated patients who survive the acute phase commonly suffer from a wide range of physical disabilities, including muscle wasting, weakness, neuromyopathies, and poor nutritional status, ${ }^{6}$ which may adversely influence the patient's clinical condition, functional status, and outcome. Rehabilitation is a non-pharmacologic therapy with the overall goal of restoring health status and reversing disability. ${ }^{7}$ The role of rehabilitation in

\footnotetext{
a Carattere Scientifico, Pavia, Italy. He is currently with the Respiratory and Critical Care Unit, Sant' Orsola-Malpighi, Bologna, Italy.

The authors have disclosed no conflicts of interest.

Correspondence: Ernesto Crisafulli MD PhD, Division of Pulmonary Rehabilitation, Ospedale Villa Pineta, Via Gaiato 127, Pavullo, Italy 41026. E-mail: crisafulli.ernesto@villapineta.it.
}

DOI: $10.4187 /$ respcare.00956 
critically ill adults remains controversial ${ }^{8}$ and not fully addressed. ${ }^{9}$

Recent randomized trials in ICU settings showed that early rehabilitation/training of mechanically ventilated patients translates into substantial functional independence at hospital discharge, both in exercise capacity ${ }^{10}$ and basic activities of daily living. ${ }^{11}$ Few non-randomized studies have addressed the role of physical training in critically ill patients on prolonged mechanical ventilation. Recently, Martin et al ${ }^{12}$ reported that the baseline performance of the upper limbs significantly correlates with the duration of mechanical ventilation. Chiang et $\mathrm{al}^{13}$ found that addition of physical training may improve functional status in patients on prolonged ventilation.

In this study we assessed changes in functional status and whether the degree of functional recovery after a comprehensive rehabilitation program influenced hospital outcomes in a population of tracheotomized and chronically ventilated patients admitted for weaning.

\section{Methods}

All the study procedures were performed according to the Declaration of Helsinki. Our hospital's ethics committee and board for clinical studies approved the study. We obtained informed written consent from all patients or their relatives.

\section{Patients}

In this prospective study, the population consisted of tracheotomized and chronically ventilated, difficult-towean patients admitted January 2006 to December 2008 to our respiratory ICU in a regional rehabilitation center (Villa Pineta Hospital, Pavullo, Italy). All the patients had been transferred from medical or surgical ICUs. The mean \pm SD onset of the acute phase was $24 \pm 3$ days before admission to our respiratory ICU. The period of ventilation via tracheotomy in the ICUs of origin was a minimum of 14 consecutive days; tracheotomy had been performed in any case as a means to facilitate weaning from mechanical ventilation.

At admission to our respiratory ICU, the patients were conscious, clinically stable, and able to adhere to the care program. The underlying clinical conditions that had caused acute respiratory failure had resolved in all the patients.

We excluded patients with persistent altered sensorium, major cardiac arrhythmia, ${ }^{9}$ history of underlying neuromuscular disease or acquired polyneuromyopathies, unable to perform physical training due to preexisting joint dysfunction, or with conditions that could have negatively influence the prognosis. We used electromyography to assess for acquired ICU neuropathy and myopathy (Fig. 1). ${ }^{14}$ Table 1 describes the subjects.

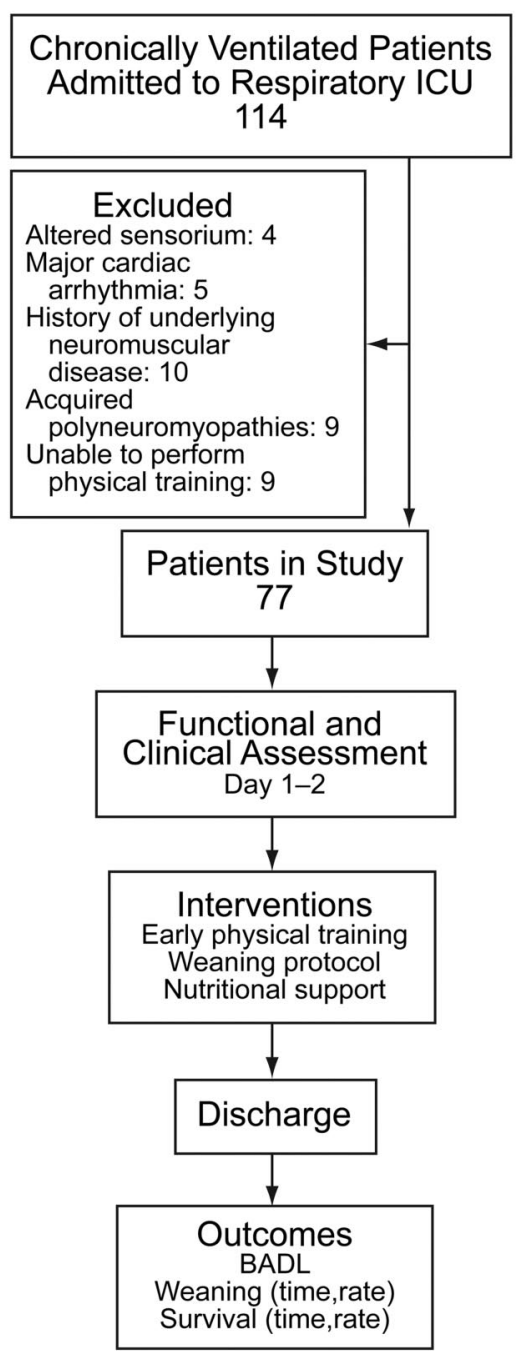

Fig. 1. Flow chart of screening, exclusion, inclusion, discharge, and outcomes. BADL = Basic Activities of Daily Living score.

\section{Care Program}

Weaning. Twenty-four hours after admission, once optimization of pharmacologic therapies and assessment of underlying conditions were verified, we performed a spontaneous breathing trial in all patients, without any mechanical assistance. None of the subjects were able to breathe spontaneously for 2 consecutive hours; all of them were on pressure-support ventilation, with the pressure-support level set according to the patient's comfort.

We followed a standardized weaning protocol, with periods of spontaneous breathing of increasing duration. ${ }^{15}$ We considered a patient successfully weaned from mechanical ventilation when they could sustain spontaneous respiration for at least 7 consecutive days.

Physical Training. Peripheral muscle training started 48 hours after admission. In the first few days this con- 
Table 1. Subjects $(n=77)$

\begin{tabular}{|c|c|}
\hline Age-y & $\begin{array}{c}75 \pm 7 \\
\text { (range } 46-88 \text { y) }\end{array}$ \\
\hline Male—no. (\%) & $46(60)$ \\
\hline Female-no. (\%) & $31(40)$ \\
\hline APACHE II—score* & $\begin{array}{c}11.5 \pm 4.4 \\
\text { (range 5.8-27.0) }\end{array}$ \\
\hline Kelly-Matthay_score $\dagger$ & $\begin{array}{c}2 \pm 1 \\
\text { (range 1-4) }\end{array}$ \\
\hline \multicolumn{2}{|l|}{ Primary Diagnosis-no. $(\%)$} \\
\hline COPD & $42(55)$ \\
\hline Restrictive disease (obesity, kyphoscoliosis) & $6(8)$ \\
\hline Heart failure & $10(13)$ \\
\hline Surgerył & $19(25)$ \\
\hline \multicolumn{2}{|l|}{ Referral Origin-no. (\%) } \\
\hline Medical ICU & $64(83)$ \\
\hline Surgical ICU & $13(17)$ \\
\hline \multicolumn{2}{|c|}{$\begin{array}{l}\text { Plus-minus values are mean } \pm \mathrm{SD} \text {. } \\
* \text { At admission to the respiratory intensive care unit. } \\
\dagger \text { Kelly-Matthay neurological status scoring system. } \\
¥ \text { Surgeries: lung, aorta, aorto-coronaric bypass, heart valve replacement. } \\
\text { APACHE = Acute Physiology and Chronic Health Evaluation } \\
\text { ICU = intensive care unit }\end{array}$} \\
\hline
\end{tabular}

sisted of active movement of the limbs, then a specific active muscular intervention consisting of trunk control, maintenance of body posture, and both upper and lower limb activities (Fig. 2) to facilitate transfer from bed to chair and standing up. As soon as possible we had the patient begin to use a wheeled walking aid (eg, rollator).

Supported or unsupported limb training was incremented daily and conducted by the same staff 6 days a week. The training period lasted a minimum of 15 sessions.

The supported training modality consisted of unloaded pedaling with a rehabilitation device (Davenbike27, Balbo Sport Ability, Dolceacqua, Italy) that allows application of various loads and positions for both leg and arm exercise with the patient in bed (see Fig. 2A) and arm cranking. The patients were instructed to maintain at least 30 revolutions per minute. Depending on the patient's ability, we progressively increased the training intensity and duration (up to an arbitrary limit of 30 min twice a day).

The unsupported training consisted of leg and arm elevations while wearing holding or wearing weights at the limb extremities (see Fig. 2 part B). We increased the loads from $100 \mathrm{~g}$ up to $500 \mathrm{~g}$ to increase the exercise intensity, and increased the frequency of 2 series to a maximum of 10 repetitions for the duration of training.

We noninvasively monitored oxygen saturation, heart rate, blood pressure, and electrocardiogram throughout both the supported and unsupported training. The physiotherapist was instructed to increase the $\mathrm{F}_{\mathrm{IO}_{2}}$ if required to maintain oxygen saturation above $92 \%$.

Physiotherapists skilled in respiratory problems, blind to the study purposes, and exclusively allocated to the respiratory ICU supervised the training and were in charge for all functional assessments. The training program was conducted independently from the weaning process. In those patients still unable to breathe spontaneously, the training activities were carried out with the aid of a portable ventilator. No specific training of the respiratory muscles was included in the training program.

Nutritional Support. The care program attention also focused on nutrition, to accomplish each patient's need during physiotherapy and according to recommendations. ${ }^{16,17}$ Daily calories was based on the estimated basal energy expenditure, calculated with the Harris-Benedict equation. ${ }^{18}$ All nutrition was provided enterally.

\section{Measurements}

We recorded anthropometry, demographics, body mass index, serum albumin, and preexisting comorbidities (via Charlson index) in the respiratory ICU at baseline. We assessed severity of illness with the Acute Physiology and Chronic Health Evaluation II $^{19}$ and consciousness with the Kelly-Matthay scale. ${ }^{20} \mathrm{We}$ also recorded pulse oximetry and calculated $\mathrm{P}_{\mathrm{aO}_{2}} / \mathrm{F}_{\mathrm{IO}_{2}}$.

We assessed respiratory muscle performance with measurements of maximum inspiratory and expiratory pressures $^{21}$ taken with a differential pressure transducer manometer (model 300, Honeywell, Freeport, Illinois), according to a previously reported method. ${ }^{22,23}$ We used the 6-point Kendall muscle testing scale to assess peripheral muscle performance of the femoral quadriceps muscles, the latissimus dorsi, and the brachial biceps muscle. ${ }^{24}$ We took the Kendall muscle testing measurements on the dominant limb, with the patient in the sitting position. We used a previously described method for the measurement on the latissimus dorsi, with the patient in lateral decubitus position. ${ }^{25}$ We used the Functional Independence Measurement scale to assess the patient's independent functioning, as previously used in a similar population. ${ }^{12}$

\section{Outcomes}

At patient discharge from the respiratory ICU we recorded survival, weaning success, and the change in Basic Activities of Daily Living (BADL) score after the rehabilitation course. ${ }^{26}$ The BADL scale is a simple 0 to 6 scale that assesses the patient's ability independently to perform bathing, clothing, moving from bed to chair, eating, evacuation, and urinary sphincter control. A binary evaluation (yes $=1$, no $=0$ ) is used to score each activity, thus contributing to the overall score. 

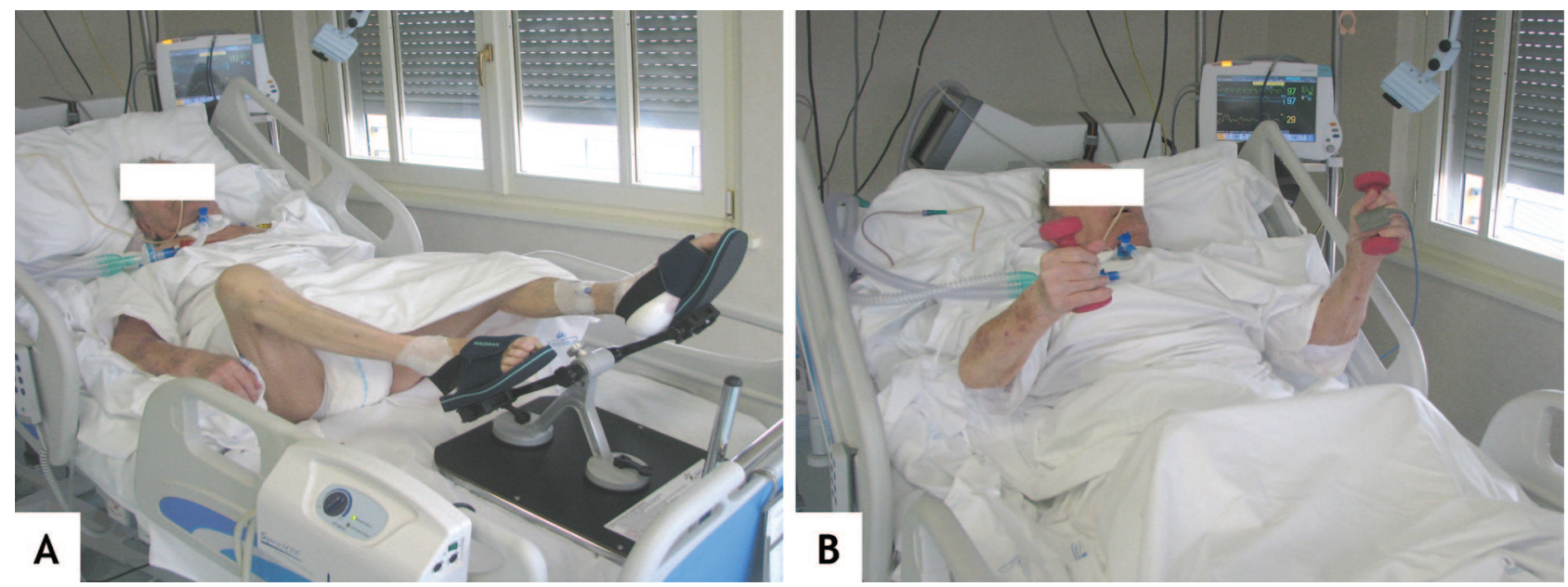

Fig. 2. Supported (A) and unsupported (B) limbs exercise during daily rehabilitation sessions.

\section{Statistical Analysis}

Analyses were carried out with statistics software (SPSS 8.0, SPSS, Chicago, Illinois). We conducted the univariate Pearson correlation analysis of the admission versus discharge change in BADL ( $\triangle \mathrm{BADL})$, respiratory ICU timing and outcomes, general characteristics, diagnosis, and cardiovascular and respiratory function at respiratory ICU admission. We then entered the parameters into a linear regression model, with $\triangle \mathrm{BADL}$ as the dependent variable, to establish their prediction power.

We then grouped the patients into $3 \triangle \mathrm{BADL}$ categories, based on the whole cohort's median $\triangle \mathrm{BADL}$ value of 2 : $\Delta \mathrm{BADL}=0 ; \Delta \mathrm{BADL} 1-2$ points; and $\triangle \mathrm{BADL}>2$ points. We calculated a survival-time analysis with log-rank, mean, and median and standard error, and graphed a KaplanMeier curve for each category to determine the cumulative probabilities of being liberated from mechanical ventilation and survival during respiratory ICU stay. In all analyses we considered an alpha $<.05$ statistically significant.

\section{Results}

The patients enrolled represented $66 \%$ of the total number of patients admitted to the respiratory ICU in the study period. The patients came from medical ICUs $(83 \%)$ and surgical ICUs (17\%) (see Table 1). COPD was the most prevalent primary diagnosis. Respiratory complications after cardiothoracic surgery and leading to difficult weaning accounted for $25 \%$ of the subjects. The mean onset of acute respiratory failure was 24 days (range 18-29 d), the mean tracheotomy day was day 16 (range day 12-21), and the mean ICU stay prior to admission to our respiratory ICU was 24 days (range 20-32 days).

Table 2 shows the cohort's status on admission (ie, measured within $48 \mathrm{~h}$ of admission). Overall, their base-
Table 2. Measurements on Admission to the Respiratory Intensive Care Unit $(n=77)$

\begin{tabular}{|c|c|c|}
\hline & Mean \pm SD & Range \\
\hline Maximum inspiratory pressure $\left(\mathrm{cm} \mathrm{H}_{2} \mathrm{O}\right)$ & $23 \pm 9$ & $10-41$ \\
\hline Maximum expiratory pressure $\left(\mathrm{cm} \mathrm{H}_{2} \mathrm{O}\right)$ & $30 \pm 12$ & $2-70$ \\
\hline Body mass index $\left(\mathrm{kg} / \mathrm{m}^{2}\right)$ & $24.8 \pm 5.2$ & $14.8-38.9$ \\
\hline Serum albumin $(g / d L)$ & $3.0 \pm 0.3$ & $2.0-4.0$ \\
\hline $\mathrm{P}_{\mathrm{aO}_{2}} / \mathrm{F}_{\mathrm{IO}_{2}}$ & $191 \pm 68$ & $90-425$ \\
\hline \multicolumn{3}{|l|}{ Blood Pressure } \\
\hline Systolic (mm Hg) & $137 \pm 18$ & $91-160$ \\
\hline Diastolic (mm Hg) & $81 \pm 9$ & $60-98$ \\
\hline Heart rate (beats/min) & $82 \pm 12$ & $63-110$ \\
\hline Charlson comorbidity index score* & $6 \pm 2$ & $2-9$ \\
\hline Basic Activities of Daily Living score $\dagger$ & $0.4 \pm 0.6$ & $0-4$ \\
\hline Functional Independence Measurement scoreł & $1.1 \pm 0.4$ & $1-5$ \\
\hline \multicolumn{3}{|l|}{ Kendall score§ } \\
\hline Femoral quadriceps muscle & $2.6 \pm 0.7$ & $0-4$ \\
\hline Brachial bicipitis muscle & $2.7 \pm 0.7$ & $0-4$ \\
\hline Broadest muscle of the back & $2.3 \pm 0.8$ & $0-4$ \\
\hline \multicolumn{3}{|c|}{$\begin{array}{l}\text { * Age uncorrected for Charlson comorbidity index. } \\
\dagger \text { The Basic Activities of Daily Living scale has a } 0-6 \text { range. } \\
\ddagger \text { The Functional Independence Measurement scale has a } 1-7 \text { range. } \\
\S \text { The Kendall score has a } 0-5 \text { range. }\end{array}$} \\
\hline
\end{tabular}

line peripheral and respiratory muscle performance, BADL, and Functional Independence Measurement scores were very low. The mean stay in our respiratory ICU was 51 days (range 12-115 d). Overall, the weaning-success and hospital-survival rates were $74 \%$ and $87 \%$, respectively.

None of the patients had an adverse event related to physical activities during the rehabilitation sessions. The mean $\pm \mathrm{SD} \triangle \mathrm{BADL}$ was $2.5 \pm 2.0$ points for the whole cohort. A large percentage of the patients who survived had recovered in all the BADL domains at respiratory ICU discharge (Fig. 3). 


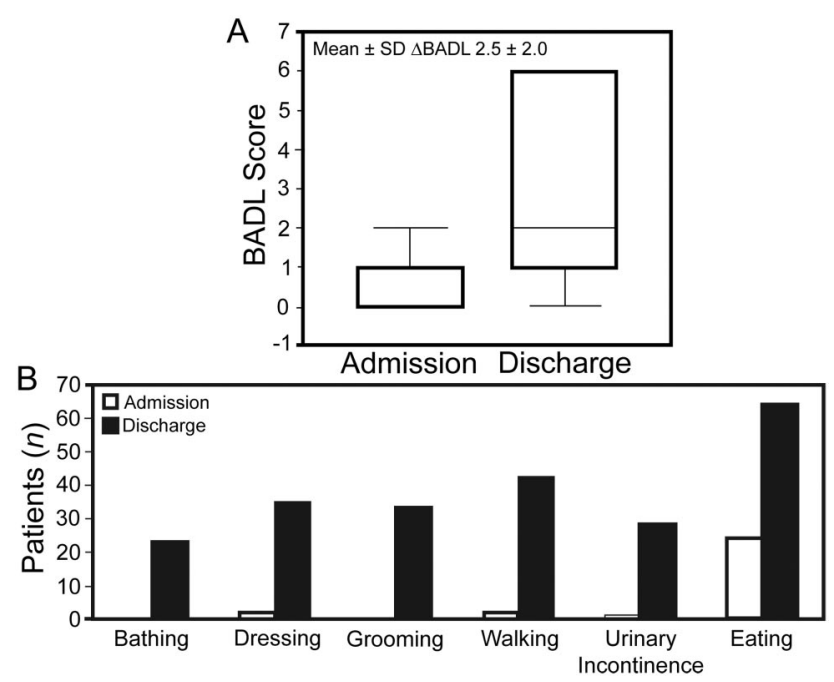

Fig. 3. A: Change in overall Basic Activities of Daily Living score ( $\triangle \mathrm{BADL}$ ) between admission and discharge. B: Changes in the 6 BADL domains between admission and discharge.

Table 3. Pearson Correlation Coefficients for Change in Basic Activities of Daily Living Score Versus Measurements at Admission

\begin{tabular}{lcc}
\hline \hline & $\mathrm{r}$ & $P$ \\
\hline Maximum inspiratory pressure & 0.033 & .41 \\
Maximum expiratory pressure & 0.078 & .30 \\
Body mass index & -0.150 & .15 \\
Serum albumin & -0.117 & .21 \\
$\mathrm{P}_{\mathrm{aO}_{2}} / \mathrm{F}_{\mathrm{IO}_{2}}$ & -0.082 & .30 \\
$\mathrm{Blood}$ pressure & & \\
$\quad$ Systolic & -0.218 & .09 \\
$\quad$ Diastolic & -0.068 & .32 \\
Heart rate & -0.011 & .47 \\
Charlson comorbidity index & 0.049 & .37 \\
Basic Activities of Daily Living score & 0.164 & .13 \\
Functional Independence Measurement scale & -0.060 & .34 \\
Kendall score & & \\
$\quad$ Femoral quadriceps muscle & 0.270 & .03 \\
Brachial bicipitis muscle & 0.238 & .05 \\
Broadest muscle of the back & 0.395 & .002 \\
\hline
\end{tabular}

Table 3 shows the Pearson correlation coefficients between $\triangle \mathrm{BADL}$ and the admission measurements. Table 4 shows the linear regression analysis for $\triangle \mathrm{BADL}$ versus the Kendall muscle testing scores. The Kendall score for the latissimus dorsi was the only variable that was significantly associated with $\triangle$ BADL $(P=.03)$.

Figure 4 shows the Kaplan-Meier curves for the 3 $\triangle$ BADL groups. There was a statistically significant difference in survival and weaning rate between the groups: the group with the lowest $\triangle \mathrm{BADL}$ had the worst clinical outcome.
Table 4. Linear Regression Analysis of Change in Basic Activities of Daily Living Score Versus Kendall Scores

\begin{tabular}{lrrrrr}
\hline \hline \multicolumn{1}{c}{ Independent Variable* } & $\beta$ & $\begin{array}{c}\text { Standard } \\
\text { Error }\end{array}$ & 95\% CI & $P$ \\
\hline Femoral quadriceps muscle & 0.021 & 0.451 & -0.845 to 0.951 & .91 \\
Brachial bicipitis muscle & -0.018 & 0.444 & -0.931 to 0.837 & .92 \\
Broadest muscle of the back & 0.388 & 0.389 & 0.111 to 1.664 & .03
\end{tabular}

* The dependent variable was the change in Basic Activities of Daily Living score.

There were 67 respiratory-ICU survivors, and at respiratory-ICU discharge 23 patients went home, 15 were transferred to a medical ward, and 29 were transferred to a rehabilitation unit.

\section{Discussion}

This study supports the concept that early rehabilitation, including peripheral muscle training, contributes to BADL recovery in difficult-to-wean patients. In contrast to previous studies in similar populations, ${ }^{12,13}$ this is the first study that found that the degree of improvement is associated with hospital outcomes such as survival and weaning success. The Kendall muscle testing score for the latissimus dorsi was an independent predictor of $\triangle \mathrm{BADL}$.

Limb training improves physical functioning in patients with COPD. ${ }^{27}$ Though it seems that early physical training should be helpful in mechanically ventilated patients who are confined to bed, no clear evidence has yet emerged in the literature in favor of early physical training in patients in the ICU setting. ${ }^{9}$ A randomized trial in a medical ICU found that only $13 \%$ of patients ventilated for $\leq 72$ hours were eligible for interrupting sedation and participating in whole-body rehabilitation to recover their basic activities. ${ }^{11}$ The feasibility of an early training program in the ICU to improve BADL functioning at hospital discharge was been further confirmed in medical and surgical mechanically ventilated patients, ${ }^{10}$ even during an episode of acute respiratory failure. ${ }^{28}$ Notwithstanding, deep sedation and unavailability of rehabilitation staff are major barriers to such rehabilitation programs. ${ }^{29}$

Nava was the first to assess the effectiveness of stepby-step physical training in a high-dependence respiratory ICU, in spontaneously breathing COPD patients just weaned from mechanical ventilation. ${ }^{30}$ In that controlled trial, Nava found that the application of gradual peripheral muscle training improved physical independence and performance. A recent study in a similar setting suggested that early rehabilitation activities are feasible and safe, even in mechanically ventilated patients, and can prevent or treat the neuromuscular complications of critical illness. ${ }^{31}$ Other investigations in patients requiring pro- 

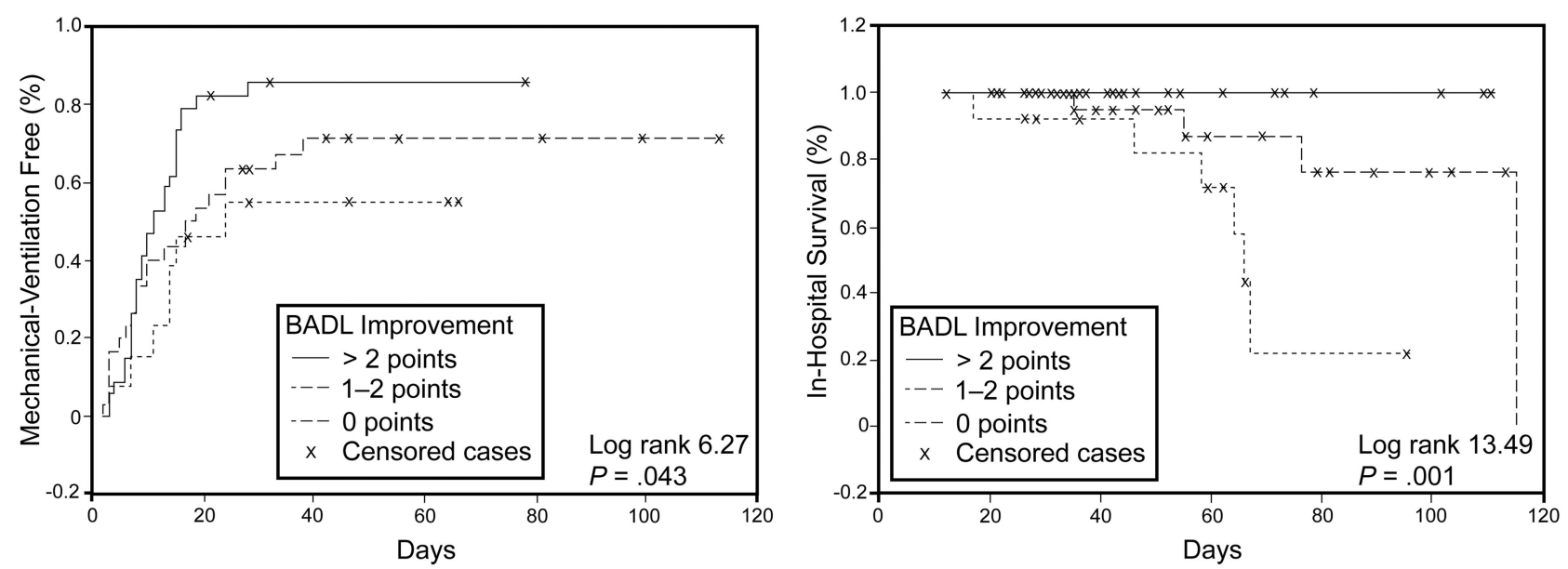

Fig. 4. Kaplan-Meier curves of the probability of being free from mechanical ventilation (left) and hospital survival (right) in the 3 categories of change in Basic Activities of Daily Living score (BADL).

longed mechanical ventilation showed that peripheral muscle strength and functional status may have both improved after a 6-week physical training period conducted by a skilled multidisciplinary team. ${ }^{12,13}$

The present study suggests that physical training can substantially improve body functionality, as assessed by the simple BADL scale (see Fig. 3), in difficult-to-wean patients in a respiratory ICU. It is noteworthy that 64 of the 77 patients improved in at least one BADL activity, and that the patients with least improvement or no change were less likely to be weaned and to live (see Fig. 4). All the patients in the $\triangle \mathrm{BADL}>2$ category survived, and over $80 \%$ of them (51\% of total) successfully weaned.

Therefore, the impact of training on BADL appears to be relevant, not only because it may be associated with the patient's recovery toward a better functional status, but also because it is likely to protect against an unfavorable long-term prognosis, as is common in critically ill patients. $^{32} \triangle \mathrm{BADL}$ following a formal rehabilitation intervention has been described first (and only) by Schweickert and colleagues, ${ }^{11}$ in patients in an ICU. The mean number of basic activities improved from 3 at ICU discharge to 6 at hospital discharge, and from 0 to 4 in the intervention and control groups, respectively, indicating that (independent of active treatment) spontaneous recovery is likely. Notwithstanding, we observed an interesting association between $\triangle \mathrm{BADL}$ following an active intervention and weaning-success and survival during stay in a critical-care area, although in a very different patient population. It is of interest, however, that hospital survival (13\%) and weaning success $(74 \%)$ were slightly better in our study than in a previous study in a population with similar characteristics. ${ }^{3}$

From a practical point of view, we can speculate that the patient's gradual progress toward autonomy (positioning, standing, walking, feeding, and bathing) with increased capacity to support the trunk, may have had important implications in weaning success. Indeed, immobility and mechanical ventilation profoundly alter the respiratory and peripheral muscles, which delays extubation and prolongs mechanical ventilation. ${ }^{6,33}$ Furthermore, this is a interesting practical result, which also made possible a direct discharge to home in around one third of our cohort, which is known to present several complications in the long-term management of care. ${ }^{33,34}$

Our study shows that, among all the characteristics we measured at admission, the Kendall muscle testing of the latissimus dorsi was the only measure that specifically predicts $\triangle \mathrm{BADL}$ after training. This finding merits consideration and interpretation. Trunk control and the ability of the upper limbs to perform BADLs may indirectly reflect the improvement of the muscle performance in the upper part of the body, which might have enabled subjects to better succeed the difficult weaning process. In an observational study, Martin et al ${ }^{12}$ found a significant correlation ( $\mathrm{r}=0.72, P<.001)$ between upper-body muscle strength at admission and shorter weaning. Nonetheless, the performance of the latissimus dorsi does not specifically or necessarily refer to the upper-limb performance, which is known to be sensitive to change after training in tracheotomized in-patients after weaning has succeeded. ${ }^{35}$ As a matter of fact, performance of any other peripheral or respiratory muscle group, or the global independence measure (which is function of the individual's muscle capacity), did not enter the prediction equation (see Table 3 ). Moreover, none of the other pre-morbid available characteristics and/or conditions of the patients at admission entered the equation either.

\section{Limitations}

This was a single-center observational study with a small sample size and no control group. Since it may be difficult 
to separate the effects of the comprehensive rehabilitation program from the natural healing and recovery, we cannot conclude that the rehabilitation intervention sped recovery or modified the hospital prognosis. Assessment of peripheral muscle performance is subjective, even if assessed by an expert physiotherapist unaware of the study purposes, and the assessment method is relatively simple. The association we observed between $\triangle \mathrm{BADL}$ and survival and weaning may not apply to patients in different settings or patients with different diagnoses. Finally, other BADL assessment instruments (eg, Barthel index as an overall function score) were not taken into account.

\section{Conclusions}

Most patients on long-term mechanical ventilation may benefit from a comprehensive rehabilitation program in a dedicated weaning center. Our findings suggest that $\triangle \mathrm{BADL}$ is associated with survival and weaning-success, and that the Kendall muscle testing performance of the latissimus dorsi is an independent predictor of $\triangle \mathrm{BADL}$.

Taking all the limitations into account, this study warrants future research with controlled trials in this area. Indeed, the application of an early intervention, including peripheral muscle training, in tracheotomized patients admitted to a respiratory ICU is of particular interest for patients who have to recover from immobility, dysfunction, and over-medication (such as with sedatives), which are typical in patients transferred from the ICU. ${ }^{36}$

\section{ACKNOWLEDGMENTS}

We thank Jessie Cross, freelance editor, for editing, and Roberto D'Amico, Biostatistics Unit, University of Modena. Italy, for advice on statistics.

\section{REFERENCES}

1. Corrado A, Roussos C, Ambrosino N, Confalonieri M, Cuvelier A, Elliott M, et al; European Respiratory Society Task Force on epidemiology of respiratory intermediate care in Europe. Respiratory intermediate care units: a European survey. Eur Respir J 2002;20(5): 1343-1350.

2. Stoller JK, Xu M, Mascha E, Rice R. Long-term outcomes for patients discharged from a long-term hospital-based weaning unit. Chest 2003;124(5):1892-1899.

3. Schönhofer B, Euteneuer S, Nava S, Suchi S, Köhler D. Survival of mechanically ventilated patients admitted to a specialised weaning centre. Intensive Care Med 2002;28(7):908-916.

4. Engoren M, Arslanian-Engoren C, Fenn-Budered N. Hospital and long-term outcome after tracheostomy for respiratory failure. Chest 2004;125(1):220-227.

5. Cox CE, Carson SE, Govert JA, Chelluri L, Sanders GD. An economic evaluation of prolonged mechanical ventilation. Crit Care Med 2007;35(8):1918-1927.
6. Topp R, Ditmyer M, King K, Doherty K, Hornyak J. The effect of bed rest and potential of rehabilitation on patients in the Intensive Care Unit. AACN Clinical Issues 2002;13(2):263-276.

7. World Health Organization. International classification of functioning, disability, and health (ICF). Geneva: World Health Organization; 2001. http://www.who.int/classifications/icf/en. Accessed December 28, 2010.

8. Clini E, Ambrosino N. Early physiotherapy in the respiratory intensive care unit. Respir Med 2005;99(9):1096-1104.

9. Gosselink R, Bott J, Johnson, Dean E, Nava S, Norrenberg M, et al; Task Force on Physiotherapy for Critically Ill Patients. Physiotherapy for adult patients with critical illness: recommendations of the European Respiratory Society and European Society of Intensive Care Medicine. Intensive Care Med 2008;34(7):1188-1199.

10. Burtin C, Clerckx B, Robbeets C, Ferdinande P, Langer D, Troosters $\mathrm{T}$, et al. Early exercise in critically ill patients enhances short-term functional recovery. Crit Care Med 2009;37(9):2499-2505.

11. Schweickert WD, Pohlman MC, Pohlman AS, Nigos C, Pawlik AJ, Esbrook CL, et al. Early physical and occupational therapy in mechanically ventilated, critically ill patients: a randomised controlled trial. Lancet 2009;373(9678):1874-1882.

12. Martin UJ, Hincapie L, Nimchuk M, Gaughan J, Criner GJ. Impact of whole-body rehabilitation in patients receiving chronic mechanical ventilation. Crit Care Med 2005;33(10):2259-2265.

13. Chiang LL, Wang LY, Wu CP, Wu HD, Wu YT. Effects of physical training on functional status in patients with prolonged mechanical ventilation. Phys Ther 2006;86(9):1271-1281.

14. Ricks E. Critical illness polyneuropathy and myopathy: a review of evidence and the implications for weaning from mechanical ventilation and rehabilitation. Physiother 2007;93:151-156.

15. Vitacca M, Vianello A, Colombo D, Clini E, Porta R, Bianchi L, et al. Comparison of two methods for weaning COPD patients requiring mechanical ventilation for more than 15 days. Am J Respir Crit Care Med 2001;164(2):225-230.

16. SINPE Consensus Paper. Glutamitaly 2003. Riv It Nutriz Parenter Enter 2004;22:115-133.

17. Kreymann KG, Berger MM, Deutz NE, Hiesmayr M, Jolliet P, Kazandjiev G, et al; European Society for Parenteral and Enteral Nutrition. ESPEN Guidelines on enteral nutrition: intensive care. Clin Nutr 2006;25(2):210-223.

18. Harris JA, Benedict FG. A biometric study of human basal metabolism. Proc Natl Acad Sci USA 1918;4(12):370-3.

19. Knaus WA, Draper EA, Wagner DP, Zimmerman JE. APACHE II: a severity of disease classification system. Crit Care Med 1985; 13(10):818-829.

20. Kelly BJ, Matthay HD. Prevalence and severity of neurological dysfunction in critically ill patients: influence on need for continued mechanical ventilation. Chest 1993;104(6):1818-1824.

21. Black L, Hyatt R. Maximal airway pressures: normal values and relationship to age and sex. Am Rev Respir Dis 1969;99(5):696-702.

22. American Thoracic Society/European Respiratory Society. ATS/ERS statement on respiratory muscle testing. Am J Respir Crit Care Med 2002;166(4):518-624.

23. Vitacca M, Paneroni M, Bianchi L, Clini E, Vianello A, Ceriana P, et al. Maximal inspiratory and expiratory pressure measurement in tracheotomised patients. Eur Respir J 2006;27(2):343-349.

24. Kendall FP, Kendall-McCreary E, Provance P. Muscles: testing and function, 4th edition. London: Lippincott, Williams \& Wilkins; 1993.

25. Lacote M, Chevalier M, Miranda AM, Bleton JP, Stevenin P. evaluation clinique de la fonction musculaire. Paris: Maloine Editeur; 1982.

26. Katz S, Ford AB, Moskowitz RW, Jackson BA, Jaffe MW. Studies of illness in the aged, the index of ADL: a standardized measure of biological and psychosocial function. JAMA 1963;185:914-919. 
27. Troosters T, Casaburi R, Gosselink R, Decramer M. Pulmonary rehabilitation in chronic obstructive pulmonary disease. Am J Respir Crit Care Med 2005;172(1):19-38.

28. Morris PE, Goad A, Thompson C, Taylor K, Harry B, Passmore L, et al. Early intensive care unit mobility therapy in the treatment of acute respiratory failure. Crit Care Med 2008;36(8):2238-2246.

29. Zanni JM, Korupolu R, Fan E, Pradhan P, Janjua K, Palmer JB, et al. Rehabilitation therapy and outcomes in acute respiratory failure: an observational pilot project. J Crit Care 2010;25(2):254-262.

30. Nava S. Rehabilitation of patients admitted to a respiratory intensive care unit. Arch Phys Med Rehabil 1998;79(7):849-854.

31. Bailey P, Thomsen GE, Spuhler VJ, Blair R, Jewkes J, Bezdjian L, et al. Early activity is feasible and safe in respiratory failure patients. Crit Care Med 2007;35(1):139-145.

32. Connors AF Jr, Dawson NV, Thomas C, Harrell FE Jr, Desbiens N, Fulkerson WJ, et al; SUPPORT investigators (Study to Understand Prognoses and Preferences for Outcomes and Risks of Treatments).
Outcomes following acute exacerbation of severe chronic obstructive lung disease. Am J Respir Crit Care Med 1996;154(4 Pt 1):959967. Erratum in: Am J Respir Crit Care Med 1997;155(1):386.

33. De Jonghe B, Bastuji-Garin S, Durand MC, Malissin I, Rodrigues P, Cerf C, et al; Groupe de Réflexion et d'Etude des Neuromyopathies en Réanimation. Respiratory weakness is associated with limb weakness and delayed weaning in critical illness. Crit Care Med 2007; 35(9):2007-2015

34. Aboussouan LS, Lattin CD, Anne VV. Determinants of time-to-weaning in a specialized respiratory care unit. Chest 2005;128(5):3117-3126.

35. Porta R, Vitacca M, Gilè LS, Clini E, Bianchi L, Zanotti E, Ambrosino N. Supported arm training in patients recently weaned from mechanical ventilation. Chest 2005;128(4):2511-2520.

36. Thomsen GE, Snow GL, Rodriguez L, Hopkins RO. Patients with respiratory failure increase ambulation after transfer to an intensive care unit where early activity is a priority. Crit Care Med 2008;36(4): 1119-1124. 